\title{
Fractional DC/DC Converter in Solar-Powered Electrical Generation Systems
}

\author{
Rubén Martínez, Yolanda Bolea and Antoni Grau \\ Automatic Control Dept. \\ Technical University of Catalonia \\ Barcelona, Spain. \\ \{ruben.martinez.gonzalez,yolanda.bolea\}@upc.edu \\ antoni.grau@upc.edu
}

\author{
Herminio Martínez \\ Electronics Engineering Department \\ Technical University of Catalonia \\ Barcelona, Spain. \\ herminio.martinez@upc.edu
}

\begin{abstract}
This paper deals with the fractional modeling of a $D C-D C$ buck-boost converter, suitable in solar-powered electrical generation systems, and the design of a fractional controller for the aforementioned switching converter. Although the modeling and design of the controller is carried out for this particular DC-DC converter, it can be easily extended to other kind of switching converter. In addition, the comparison between integer-order plant/controller and fractional-order plants/controller is carried out. The article also shows that, under the same design conditions, the fractional-order controller has a better performance and behaviour than the classical integer-order controller in both situations, that is, with integer-order plant and fractional-order plant models.
\end{abstract}

\section{Introduction}

The finite global supply of recoverable fossil fuels implies that at some point in the future, alternative sources of energy will become the primary source of energy to meet global demand. Solar cells represent promising alternative that will likely initially supplement fossil fuel based energy supply, and eventually replace the fossil fuel energy sources as the availability of the latter decline.

Photovoltaic (PV) arrays are generally the bulkiest and most expensive parts of solar-powered electrical generation systems. Optimum utilization of available power from these arrays is therefore essential and can considerably reduce the size, weight and cost of such power systems. The controller is usually an essential part of a PV system as shown in Figure 1.

The controller incorporates a DC-DC converter and is used as a controlled energy-transfer-equipment between the main energy source (PV arrays) and an auxiliary energy system based on ultracapacitors. Most converters are based on either the buck converter (step-down), boost convert (step-up) or buck-boost converter setup. This capability of the converter makes it ideal for converting the solar panel maximum power point voltage to the load operating voltage [1]. Problems exist with battery packs including

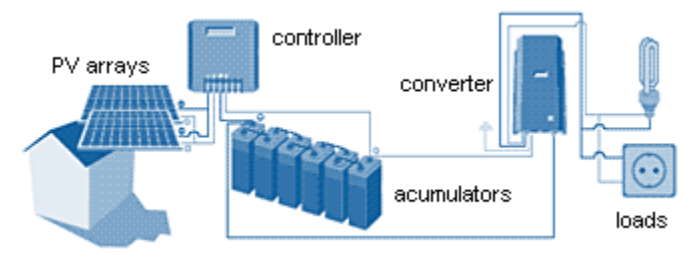

Figure 1. Diagram of an isolated solarpowered system.

the inability to absorb and discharge large current loads during regenerative braking and boost assist, performance degradation over their life, weight, size and environmental concerns regarding disposal. Ultracapacitors, or electrochemical capacitors (EC), can eliminate these problems.

The performance characteristics of ultracapacitors differ somewhat from those of conventional capacitors. The impedance of any real ultracapacitor can be easily reproduced in any frequency model equation by replacing every $j w$ expression with $(j w)^{\alpha}, 0<\alpha<1$, and where $\alpha=1$ represents an ideal capacitor with no frequency dependence [3]. Experimentally, the parameter $\alpha$ is not often smaller than 0.5 , the case for a Warburg impedance. A single value of $\alpha$ normally describes an electrochemical system over only a limited frequency range [4].

This non-ideality is a typical feature of electrochemical charging processes, and may be interpreted as resulting from a distribution in macroscopic path lengths (nonuniform active layer thickness) or a distribution in microscopic charge transfer rates, absorption processes, or surface roughness [3]. For distributed parameter systems, it has been shown that fractional order calculus will play a role in its modeling and analysis. In general, fractionalorder systems are useful to model various stable physical phenomena (commonly diffusive systems) with anomalous decay, say those that are not of exponential type.

It is natural to consider fractional order controls. Clearly, for closed-loop control systems, there are four situations. They are: 1) IO (integer order) plant with IO con- 
troller; 2) IO plant with FO (fractional order) controller; 3) FO plant with IO controller and 4) FO plant with FO controller.

In this paper, we focus on the control of a Buck-Boost converter based on ultracapacitors as an essential element in the optimal use of available energy in the PV arrays. A fractional control approach is motivated by the fractional nature that presents the model of the converter with ultracapacitors as accumulator. Furthermore, FO and IO linear feedback controllers are designed and compared in the control of the FO and IO models that can describe the plant in different frequency ranges.

\section{A Survey of Fractional Calculus}

The idea of non-integer order derivatives is as old as regular calculus. Fractional calculus has been used for modeling different physical phenomena [6] and in control theory ( $[10]$; [11]; [12]). We can notice systems in nature with fractional behaviour, but many of them with a very low fractionality [13].

The fractional integral operator is defined by [6]

$$
I_{t}^{\alpha} f(t)=\frac{1}{\Gamma(\alpha)} \int_{0}^{t}(t-\tau)^{\alpha-1} f(\tau) d \tau
$$

and we adopt Caputo definition for fractional derivative of order $\alpha$ of any function $f(t)$ :

$$
\begin{gathered}
D^{\alpha} f(t)=I^{n-\alpha} D^{n} f(t) \triangleq \frac{1}{\Gamma(n-\alpha)} \int_{0}^{t} \frac{f^{(n)(\tau)}}{(t-\tau)^{\alpha-n+1}} d \tau \\
n-1<\alpha<n, \quad \alpha \in \mathbb{R}^{+}
\end{gathered}
$$

where gamma function $\Gamma(\nu)$ is defined for $\nu>0$ as:

$$
\Gamma(\nu)=\int_{0}^{\infty} x^{\nu-1} e^{-x} d x
$$

\section{State-Space averaging model of an ideal Buck-Boost converter based on ultraca- pacitors}

Many scientists have worked in order to obtain different capacitor models, Westerlund and Ekstam (see in [7]) proposed that better capacitor impedance could be

$$
Z(j w)=\frac{1}{(j w)^{\alpha} C} ; 0<\alpha<1
$$

Based on the last expression, current $i(t)$ across the capacitor is a function of a general voltage $v(t)$

$$
i(t)=C D_{t}^{\alpha} v(t)
$$

It can be noticed that, $D_{t}^{\alpha} v(t)$ is the fractional time derivative of voltage. For different capacitors $\alpha$ is different to one, and is close to 0.999 . Figure 2 displays the Nyquist diagram for the capacitor models (real and ideal).
According to some papers, ultracapacitors can be modeled by zones where at low frequencies are similar to classical capacitors $(\alpha \approx 1)$ and at medium frequencies are characterized by diffusion effect. Furthermore, they are better characterized in the Warburg domain $(j w)^{1 / 2}$ than in the classical Laplace domain $(j w)$ [8]. At higher frequencies the resistance as well as the capacitance of a porous electrode decreases, because only part of the active porous layer is accessible at high frequencies. The ultracapacitor may thus be represented by an ideal capacitor [3].

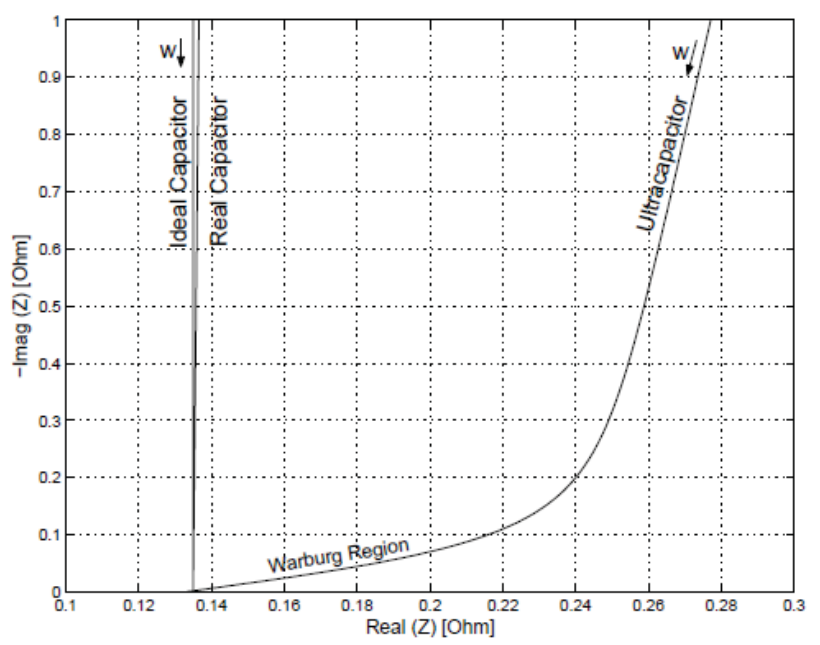

\section{Figure 2. Nyquist diagram of a capacitor (real and ideal) and an ultracapacitor.}

Let us apply the state-space averaging method to model the buck-boost converter of Figure 3. The fundamental difference of this class of converter with the Buck and Boost converters is that the output voltage has an opposite sign to DC source $E(t)$. The input voltage $E(t)$ is an independent source whose value is defined by the MPPT (maximum power point tracking) of a PV system.

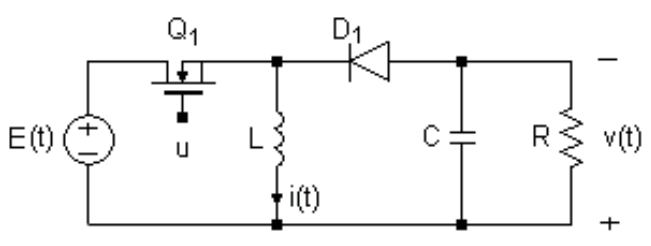

Figure 3. Buck-Boost converter system.

The operation of this system is as follows: when the transistor $Q_{1}$ is switched to ON state (conduction state), the diode $D_{1}$ is inversely polarized generating a circuit topology which is shown in Figure 4. During this period, the inductor current is generated from the source $E(t)$. While the diode remains inversely polarized we say the circuit is operating in the "charging period".

When the transistor is switched OFF, the diode is directly polarized generating the circuit topology shown in 
Figure 5. This second period is known as the "discharging period" due to the fact that the stored energy in the inductor $L$ is transferred to the system load $R$ [5].

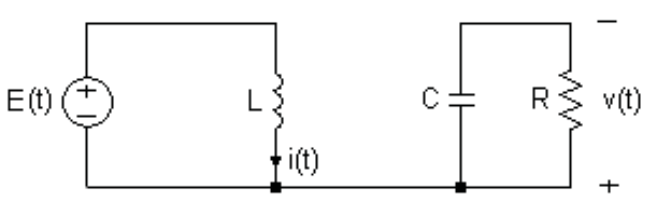

Figure 4. Switch position at $u=1$.

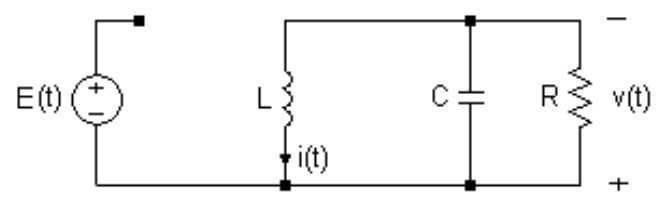

Figure 5. Switch position at $u=0$.

When Kirchoff's voltage and current laws are applied to both circuit topologies of Figures 4-5, and the obtained models are combined into a single dynamic model, the resulting system of differential equations describing the Buck-Boost converter is the following:

$$
\begin{aligned}
L D_{t}^{1} i(t) & =(1-u) v(t)+u E(t) \\
C D_{t}^{\alpha(w)} v(t) & =-\frac{1}{R} v(t)-(1-u) i(t)
\end{aligned}
$$

where $\alpha(w)=\{1,0.5,1\}$ describes the electrochemical system over a frequency range $w$ (low, medium and high frequency, respectively). The normalized average model of the ideal Buck-Boost converter based on ultracapacitors is given by

$$
\begin{aligned}
& D_{\tau}^{1} x_{1}=u_{x} x_{2}+\left(1-u_{x}\right) E \\
& D_{\tau}^{\alpha(w)} x_{2}=-u_{x} x_{1}-\frac{1}{Q} x_{2}
\end{aligned}
$$

where the variable $x_{1}$ represents the normalized inductor current, $x_{2}$ is the normalized output voltage and $u_{x}=$ $1-u$ represents the average control variable. Clearly the underlying transformation is given by

$$
\begin{gathered}
{\left[\begin{array}{l}
x_{1} \\
x_{2}
\end{array}\right]=\left[\begin{array}{cc}
\sqrt{\frac{L}{C}} & 0 \\
0 & 1
\end{array}\right]\left[\begin{array}{l}
i \\
v
\end{array}\right]} \\
Q=R \sqrt{\frac{C}{L}}, \quad \tau=\frac{t}{\sqrt{L C}}
\end{gathered}
$$

\section{State Feedback Controller Design}

Let us consider the tangent linearization model of the normalized average ideal Buck-Boost converter system defined by (5) and around the equilibrium point:

$$
z_{2}=v^{*}<0 ; z_{1}=-\frac{v^{*}\left(E-v^{*}\right)}{Q E} ; u_{z}=\frac{E}{E-v^{*}}
$$

where $v^{*}$ is the normalized reference voltage. The linearization of the average model is given by

$$
\begin{gathered}
D_{\tau}^{1} e_{1}=\frac{E}{\left(E-v^{*}\right)} e_{2}-\left(E-v^{*}\right) u_{e} \\
D_{\tau}^{\alpha(w)} e_{2}=-\frac{E}{E-v^{*}} e_{1}-\frac{1}{Q} e_{2}+\frac{v^{*}\left(E-v^{*}\right)}{Q E} u_{e}
\end{gathered}
$$

where

$$
\begin{gathered}
e_{1}=x_{1}-z_{1}, \quad e_{2}=x_{2}-z_{2}, \\
u_{e}=u_{x}-u_{z}
\end{gathered}
$$

The objective is to find an stabilizing control law $u_{e}(t)$ such as:

1. The equilibrium point $e=0$ of (6) is locally and asymptotically stable.

2. The control system must reject constant disturbances, like:

$$
\lim _{t \rightarrow \infty}\left[v(t)-v^{*}(t)\right]=0
$$

3. $0 \leq u_{x}(t) \leq 1, \forall t \geq 0$.

4. The eigenvalues of the average feedback state can be arbitrarily assigned.

\subsection{IO Controller}

In this case, an average integer and linear state feedback control is sought in the form:

$$
u_{e}=-k_{1} I_{\tau}^{1} e_{1}-k_{2} e_{1}-k_{3} e_{2}
$$

which drives the average stabilization error state $e$ to zero in an exponentially stable manner. Such a controller is designed with the help of the average tangent linearization system and it will use, for the average nonlinear system, the control input

$$
u_{x}=u_{z}-k_{1} I_{\tau}^{1} e_{1}-k_{2} e_{1}-k_{3} e_{2}
$$

IO Plant (low and high frequencies)

The equivalent closed loop tangent system for $\alpha(w)=1$ is given by

$$
\begin{gathered}
D_{\tau}^{2} e_{1}=R k_{2} D_{\tau}^{1} e_{1}+R k_{1} e_{1}+\left[R k_{3}+\frac{E}{R}\right] D_{\tau}^{1} e_{2} \\
D_{\tau}^{2} e_{2}=-\left[P k_{3}+\frac{1}{Q}\right] D_{\tau}^{1} e_{2}-\left[P k_{2}+\frac{E}{R}\right] D_{\tau}^{1} e_{1}-P k_{1} e_{1}
\end{gathered}
$$

where $P=\frac{v^{*} R}{Q E}$ and $R=\left(E-v^{*}\right)$. In matrix form is expressed as:

$$
D_{\tau}^{1} e_{i o i o}=\left[\begin{array}{ccc}
0 & 1 & 0 \\
h_{1} & h_{2} & h_{3} \\
h_{4} & h_{5} & h_{6}
\end{array}\right] e_{\text {ioio }}
$$

where 


$$
\begin{aligned}
& h_{1}=R k_{1}, h_{2}=R k_{2} \\
& h_{3}=R k_{3}+\frac{E}{R}, h_{4}=-P k_{1} \\
& h_{5}=-\left(P k_{2}+\frac{E}{R}\right), h_{6}=-\left(P k_{3}+\frac{1}{Q}\right)
\end{aligned}
$$

$e_{\text {ioio }}=\left[e_{1}, D_{\tau}^{1} e_{1}, D_{\tau}^{1} e_{2}\right]^{T}$ and its characteristic polynomial is given by

$$
p(s)=s^{3}+a_{1 i o} s^{2}+a_{2 i o} s+a_{3 i o} s
$$

for

$$
\begin{aligned}
& a_{1 i o}=P k_{3}-R k_{2}+1 / Q \\
& a_{2 i o}=E k_{3}+\left(\frac{E}{R}\right)^{2}+\frac{\left(2 v^{*}-E\right)}{Q} k_{2}-R k_{1} \\
& a_{3 i o}=\frac{\left(2 v^{*}-E\right)}{Q} k_{1}
\end{aligned}
$$

FO plant (medium frequencies)

The equivalent closed loop tangent system for $\alpha(w)=$ 0.5 is given by

$$
D_{\tau}^{2} e_{1}=R k_{2} D_{\tau}^{1} e_{1}+R k_{1} e_{1}+\left[R k_{3}+\frac{E}{R}\right] D_{\tau}^{1} e_{2}
$$

$D_{\tau}^{1.5} e_{2}=-\left[P k_{3}+\frac{1}{Q}\right] D_{\tau}^{1} e_{2}-\left[P k_{2}+\frac{E}{R}\right] D_{\tau}^{1} e_{1}-P k_{1} e_{1}$

In matrix form is expressed as:

$$
D_{\tau}^{0.5} e_{\text {iofo }}=\left[\begin{array}{ccccc}
0 & 1 & 0 & 0 & 0 \\
0 & 0 & 1 & 0 & 0 \\
0 & 0 & 0 & 1 & 0 \\
h_{1} & 0 & h_{2} & 0 & h_{3} \\
0 & 0 & 0 & 0 & 1 \\
h_{4} & 0 & h_{5} & 0 & h_{6}
\end{array}\right] e_{i o f o}
$$

where

$$
e_{i o f o}=\left[e_{1}, D_{\tau}^{0.5} e_{1}, D_{\tau}^{1} e_{1}, D_{\tau}^{1.5} e_{1}, D_{\tau}^{1} e_{2}\right]^{T}
$$

and its characteristic polynomial is given by

$$
\begin{aligned}
& p(\lambda)=\lambda^{5}+a_{1 f o} \lambda^{4}+a_{2 f o} \lambda^{3}+a_{3 f o} \lambda^{2}+a_{4 f o} \lambda+a_{5 f o} \\
& \text { for } \lambda=s^{0.5} \text { and } \\
& a_{1 f o}=P k_{3}+\frac{1}{Q}, a_{2 f o}=-R k_{2} \\
& a_{3 f o}=E k_{3}+\frac{\left(2 v^{*}-E\right)}{Q} k_{2}+\frac{E^{2}}{R^{2}} \\
& a_{4 f o}=-R k_{1}, a_{5 f o}=\frac{\left(2 v^{*}-E\right)}{Q} k_{1}
\end{aligned}
$$

\subsection{FO Controller}

In this section, the degree of freedom yielded by fractional models in state space is used to offer fractional controllers for each plant.

IO Plant (low and high frequencies)

In this case, an average non-integer and linear state feedback control is sought in the form:

$$
u_{e}=-q_{1} I_{\tau}^{0.5} e_{1}-q_{2} e_{1}-q_{3} D_{\tau}^{0.5} e_{1}-q_{4} e_{2}-q_{5} D_{\tau}^{0.5} e_{2}
$$

which drives the average stabilization error state $e$ to zero in a generalized exponentially stable manner. Such a controller is designed with the help of the average tangent linearization system and it will use, for the average nonlinear system, the control input

$$
u_{x}=u_{z}+u_{e}
$$

The equivalent closed loop tangent system for $\alpha(w)=$ 1 is given by

$$
\begin{gathered}
D_{\tau}^{1.5} e_{1}=R q_{1} e_{1}+R q_{2} D_{\tau}^{0.5} e_{1}+R q_{3} D_{\tau}^{1} e_{1}+ \\
{\left[\frac{E}{R}+R q_{4}\right] D_{\tau}^{0.5} e_{2}+R q_{4} D_{\tau}^{1} e_{2}} \\
D_{\tau}^{1.5} e_{2}=-P q_{1} e_{1}-\left[\frac{E}{R}+P q_{2}\right] D_{\tau}^{0.5} e_{1}-P q_{3} D_{\tau}^{1} e_{1}- \\
{\left[\frac{1}{Q}+P q_{4}\right] D_{\tau}^{0.5} e_{2}-P q_{5} D_{\tau}^{1} e_{2}}
\end{gathered}
$$

In matrix form is expressed as:

$$
D_{\tau}^{0.5} e_{\text {foio }}=\left[\begin{array}{ccccc}
0 & 1 & 0 & 0 & 0 \\
0 & 0 & 1 & 0 & 0 \\
g_{1} & g_{2} & g_{3} & g_{4} & g_{5} \\
0 & 0 & 0 & 0 & 1 \\
g_{6} & g_{7} & g_{8} & g_{9} & g_{10}
\end{array}\right] e_{\text {foio }}
$$

where

$$
\begin{aligned}
& g_{1}=R q_{1}, g_{2}=R q_{2}, g_{3}=R q_{3}, g_{4}=\frac{E}{R}+R q_{4} \\
& g_{5}=R q_{5}, g_{6}=-P q_{1}, g_{7}=-\left[\frac{E}{R}+P q_{2}\right] \\
& g_{8}=-P q_{3}, g_{9}=-\left[\frac{1}{Q}+P q_{4}\right], g_{10}=P q_{5}
\end{aligned}
$$

$$
e_{\text {foio }}=\left[e_{1}, D_{\tau}^{0.5} e_{1}, D_{\tau}^{1} e_{1}, D_{\tau}^{0.5} e_{2}, D_{\tau}^{1} e_{2}\right]^{T}
$$

and its characteristic polynomial is given by

$$
p(\lambda)=\lambda^{5}+b_{1 i o} \lambda^{4}+b_{2 i o} \lambda^{3}+b_{3 i o} \lambda^{2}+b_{4 i o} \lambda+b_{5 i o}
$$

for

$$
\begin{aligned}
& b_{1 i o}=P q_{5}-R q_{3}, b_{2 i o}=P q_{4}-R q_{2}+\frac{1}{Q} \\
& b_{3 i o}=\frac{\left(2 v^{*}-E\right)}{Q} q_{3}-R q_{1}+E q_{5} \\
& b_{4 i o}=E q_{4}+\frac{E^{2}}{R^{2}}+\frac{\left(2 v^{*}-E\right)}{Q} q_{2}, b_{5 i o}=\frac{\left(2 v^{*}-E\right)}{Q} q_{1}
\end{aligned}
$$




\section{FO plant (medium frequencies)}

In this case, an average non-integer and linear state feedback control is sought in the form:

$$
u_{e}=-q_{1} I_{\tau}^{0.5} e_{1}-q_{2} e_{1}-q_{3} D_{\tau}^{0.5} e_{1}-q_{4} e_{2}
$$

which drives the average stabilization error state $e$ to zero in a generalized exponentially stable manner. Such a controller is designed with the help of the average tangent linearization system and it will use, for the average nonlinear system, the control input

$$
u_{x}=u_{z}+u_{e}
$$

The equivalent closed loop tangent system for $\alpha(w)=$ 0.5 is given by

$$
D_{\tau}^{1.5} e_{1}=R q_{1} e_{1}+R q_{2} D_{\tau}^{0.4} e_{1}+R q_{3} D_{\tau}^{1} e_{1}+
$$

$$
\left[\frac{E}{R}+R q_{4}\right] D_{\tau}^{0.5} e_{2}
$$

$D_{\tau}^{1} e_{2}=-P q_{1} e_{1}-\left[\frac{E}{R}+P q_{2}\right] D_{\tau}^{0.5} e_{1}-P q_{3} D_{\tau}^{1} e_{1}-$

$$
\left[\frac{1}{Q}+P q_{4}\right] D_{\tau}^{0.5} e_{2}
$$

In matrix form is expressed as:

$$
D_{\tau}^{0.5} e_{f o f o}=\left[\begin{array}{cccc}
0 & 1 & 0 & 0 \\
0 & 0 & 1 & 0 \\
g_{1} & g_{2} & g_{3} & g_{4} \\
g_{6} & g_{7} & g_{8} & g_{9}
\end{array}\right] e_{f o f o}
$$

where

$$
e_{f o f o}=\left[e_{1}, D_{\tau}^{0.5} e_{1}, D_{\tau}^{1} e_{1}, D_{\tau}^{0.5} e_{2}\right]^{T}
$$

and its characteristic polynomial is given by

$$
p(\lambda)=\lambda^{4}+b_{1 f_{o}} \lambda^{3}+b_{2 f_{o}} \lambda^{2}+b_{3 f_{o}} \lambda+b_{4 f_{o}}
$$

for

$$
\begin{aligned}
& b_{1 f o}=P q_{4}-R q_{3}+\frac{1}{Q} \\
& b_{2 f o}=\frac{\left(2 v^{*}-E\right)}{Q} q_{3}-R q_{2} \\
& b_{3 f o}=\frac{\left(2 v^{*}-E\right)}{Q} q_{2}-R q_{1}+E q_{4}+\frac{E^{2}}{R^{2}} \\
& b_{4 f o}=\frac{\left(2 v^{*}-E\right)}{Q} q_{1}
\end{aligned}
$$

Equating these polynomials to a desired closed loop characteristic polynomial, feedback gains for the rational linear controllers can be obtained [9].

\subsection{Simulations}

Some simulations are carried out in order to assess the effectiveness of the proposed full state feedback controllers, computed on basis of the tangent linearized systems, to accomplish a stabilization around a normalized equilibrium point value for initial conditions set at origin of coordinates.

In order to compare the performances of different control laws (IO and FO controllers for IO and FO plants), the same poles placement than in closed loop system is used for determinate the feedback gains. All zeros of the characteristic polynomial are defined by $\epsilon$.

The following parameters and design values have been used:

$$
Q=0.75, E(t)=10 V, v^{*}=-5 V, \epsilon=0.15
$$

The average control input initially takes negative values and then a slower response is proposed. This would cause a temporary saturation to zero of the corresponding switched controller. In order to verify that the control

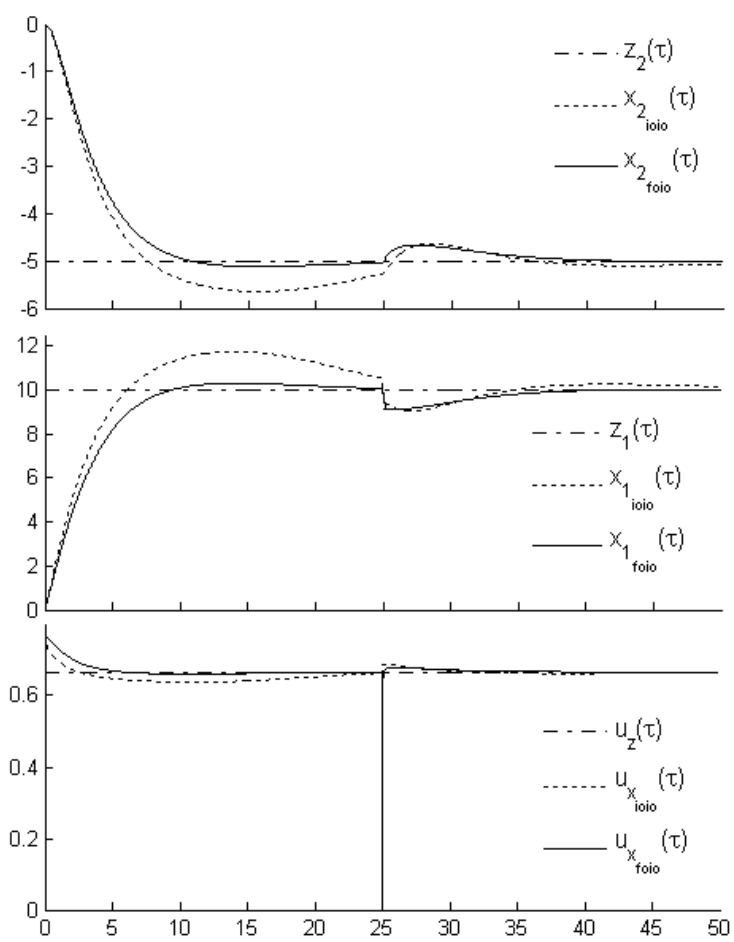

\footnotetext{
Figure 6. Response of average Buck-Boost converter based on ultracapacitors to linear state feedback controllers $(\alpha(w)=1)$.
}

system rejects constant disturbances, at $t=25 \mathrm{~s}$ a step signal is used as disturbance on output. Figure 6 depicts the response of the nonlinear average Buck-Boost converter circuit based on ultracapacitors for low frequencies to IO and FO control actions of state feedback controllers computed on the basis of the linearized tangent average system complemented with the nominal equilibrium control 


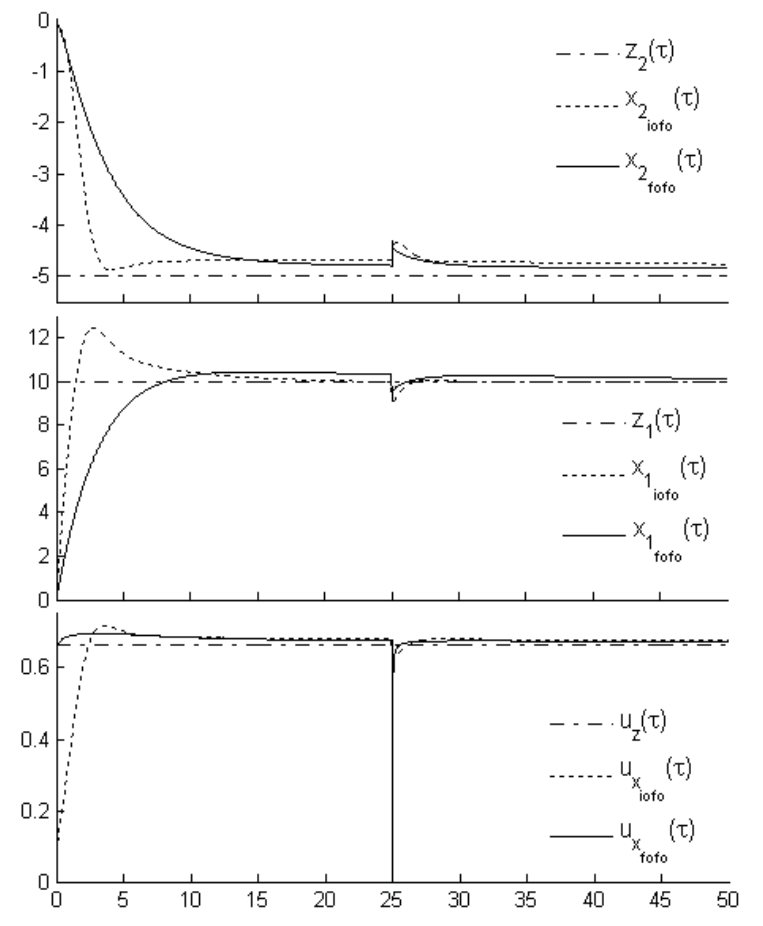

Figure 7. Response of average Buck-Boost converter based on ultracapacitors to linear state feedback controllers $(\alpha(w)=0.5)$.

input. Similarly, Figure 7 depicts the response of the nonlinear average Buck-Boost converter circuit for medium frequencies. In both plants (IO and FO plants), FO controllers show a best behaviour at closed loop system than IO controllers. The responses are smoother and the convergence to the origin is higher.

\section{Conclusions}

In this work, fractional modeling of a DC-DC buckboost converter based on ultracapacitors, suitable for many powered electrical systems, is presented. As the fractional model of the system changes according to the frequencies range, FO and $\mathrm{IO}$ models are proposed. Therefore, FO and IO linear feedback controllers are designed and compared in each plant. In simulation results is showed that FO controllers are more suitable for both plants (IO and FO plants) than IO controllers, which represents a strong motivation to the modeling and control of powered electrical systems via fractional control techniques.

\section{Acknowledgments}

Authors would like to thank Inter-Ministerial Commission of Spanish Government (DPI2004-5414, MCYT), Catalan Autonomous Government (VIS, Consolidated Research Group), European Commission (FP6-2005-IST6, URUS-045062) and Cooperation Spanish Agency for
International Development (AECID) for funding this research.

\section{References}

[1] Castaner, L., Silvestre, S., Modeling Photovoltaic Systems, Wiley, 2002.

[2] Chung, H.S.H., Ho, M.T., Hui, S.Y.R., Tse, K.K., $A$ Novel Maximum Power Point Tracking Technique for PV Panels, Power Electronics Specialist Conference, 4:1970-1975, June 2001.

[3] R. Kötz a, M. Carlen, Principles and applications of electrochemical capacitors. Electrochimica, Acta 45 (2000), 2483 - 2498.

[4] M. Sullivan, R. Kötz and O. Haas, Electrochemically Modified Glassy Carbon as an Electrochemical Capacitor Material, The Electrochemical Society Proceedings, Vol. 95-29.

[5] Sira-Ramírez, H. and Silva-Ortigoza, R., Control Design Techniques in Power Electronics Devices, Springer-Verlag, 2006.

[6] Podlubny, I., Fractional Differential equations, San Diego: Academic Press, 1999.

[7] Westerlund, S. and Ekstam, L., Capacitor theory, IEEE Transactions on Dielectrics and Electrical Insulation, vol. 1, 1994.

[8] Quintana, J. J., Ramos, A. and Nuez, I. Identification of the Fractional Impedance of Ultracapacitors, Proceedings of the 2nd IFAC Workshop on Fractional Differentiation and its Applications, Porto, Portugal, 2006.

[9] Martínez, R. and Feliu, V., Linear Control Rational Systems Approach on State Space, Symposium on Applied Fractional Calculus, Badajoz, Spain, 2007.

[10] Podlubny, I., Fractional-order systems and $P I^{\lambda} D^{\mu}$ controllers. IEEE Trans. Automatic Cont., vol. 44, no. 1, 1999, pp. $208-214$.

[11] J. A. Tenreiro Machado, Analysis and Design of Fractional-Order Digital Control Systems, Journal Systems Analysis-Modelling-Simulation, Gordon \& Breach Science Publishers, vol. 27,pp. 107 - 122, 1997.

[12] Patrick Lanusse, Alain Oustaloup, Jocelyn Sabatier, Step-By-Step Presentation of a 3rd Generation CRONE Controller Design With An Anti-Windup System, ENOC-2005, EUROMECH Nonlinear Oscillations Conference, Eindhoven, Netherlands, 2005.

[13] Torvik, P.J. and Bagley, R. L., On the Appearance of the Fractional Derivative in the Behavior of Real Materials. Transactions of the ASME, 51, 294 - 298, 1984. 